\title{
Primordial Magnetic Field Limits from Cosmic Microwave Background Bispectrum of Magnetic Passive Scalar Modes
}

\author{
Pranjal Trivedi \\ Sri Venkateswara College, University of Delhi, Delhi 110021, India \\ \& Department of Physics and Astrophysics, University of Delhi, Delhi 110007, India * \\ Kandaswamy Subramanian \\ IUCAA, Post Bag 4, Ganeshkhind, Pune 411 007, India \\ T. R. Seshadri \\ Department of Physics and Astrophysics, University of Delhi, Delhi 110007, India用
}

(Dated: September 1, 2018)

\begin{abstract}
Primordial magnetic fields lead to non-Gaussian signals in the cosmic microwave background (CMB) even at the lowest order, as magnetic stresses and the temperature anisotropy they induce depend quadratically on the magnetic field. In contrast, CMB non-Gaussianity due to inflationary scalar perturbations arises only as a higher order effect. Apart from a compensated scalar mode, stochastic primordial magnetic fields also produce scalar anisotropic stress that remains uncompensated till neutrino decoupling. This gives rise to an adiabatic-like scalar perturbation mode that evolves passively thereafter (called the passive mode). We compute the CMB reduced bispectrum $\left(b_{l_{1}} l_{2} l_{3}\right)$ induced by this passive mode, sourced via the Sachs-Wolfe effect, on large angular scales. For any configuration of bispectrum, taking a partial sum over mode-coupling terms, we find a typical value of $l_{1}\left(l_{1}+1\right) l_{3}\left(l_{3}+1\right) b_{l_{1} l_{2} l_{3}} \sim 6-9 \times 10^{-16}$, for a magnetic field of $B_{0} \sim 3 \mathrm{nG}$, assuming a nearly scale-invariant magnetic spectrum . We also evaluate, in full, the bispectrum for the squeezed collinear configuration over all angular mode-coupling terms and find $l_{1}\left(l_{1}+1\right) l_{3}\left(l_{3}+1\right) b_{l_{1}} l_{2} l_{3} \approx-1.4 \times 10^{-16}$. These values are more than $\sim 10^{6}$ times larger than the previously calculated magnetic compensated scalar mode CMB bispectrum. Observational limits on the bispectrum from WMAP7 data allow us to set upper limits of $B_{0} \sim 2 \mathrm{nG}$ on the present value of the cosmic magnetic field of primordial origin. This is over 10 times more stringent than earlier limits on $B_{0}$ based on the compensated mode bispectrum.
\end{abstract}

PACS numbers: 98.62.En, 98.70.Vc, 98.80.Cq

\section{INTRODUCTION}

The origin and evolution of large-scale magnetic fields in the universe is not yet clearly understood. There is observational evidence for micro-Gauss strength magnetic fields, ordered on kiloparsec to 10 kiloparsec scales, in galaxies and clusters of galaxies [1-5]. There is also evidence for galactic scale fields at higher redshift [6, 7]. There are only tentative indications, to date, of fields not associated with individual galaxies or clusters [8]. Further, recent $\gamma$-ray observations using Fermi/LAT data provide hints of a detection [9] or a lower bound [10] of $B \sim 10^{-16} \mathrm{G}$ on intergalactic scales. Constraints on purely cosmological magnetic fields have also been derived from the CMB [11 30], big bang nucleosynthesis and polarized radiation from extragalactic radio sources (for a review see e.g. [31]).

It is generally accepted that the observed fields require a seed magnetic field, possibly of primordial origin, which are then amplified by astrophysical processes [3, 4, 31-34]. A strong enough seed may mainly require amplification due to flux-freezing which arises during the collapse to form structures [4]. On the other hand a weak seed would require con-

\footnotetext{
* ptrivedi@physics.du.ac.in

† kandu@iucaa.ernet.in

trs@physics.du.ac.in
}

siderable dynamo action as well [3, 4, 32-34]. As yet no compelling theory exists for the origin of strong primordial fields. Equally, dynamo theories are not without their own difficulties, in particular, how large-scale dynamos lead to coherent enough fields on saturation [3]. Thus it is important to keep an open mind on the issue of the origin of magnetic fields.

Large-scale primordial fields could have resulted from phase transitions in the early universe, for example during the inflationary era [35-39]. An important way to constrain or detect such fields is via their imprints on the Cosmic Microwave Background (CMB) anisotropies. Considerable work has already been done to calculate the magnetic field signals in the power spectrum (both temperature and polarization) of the CMB anisotropies [13-30,40-45]. The possibility of non-Gaussian signals in the CMB temperature anisotropies has drawn increased attention of late. This possibility is particularly relevant when one considers magnetically induced anisotropies in the $\mathrm{CMB}$, for the following reason: In standard inflationary models, the Gaussian statistics of the quantum fluctuations in the inflaton field leads to Gaussian statistics for the CMB temperature field. In such models, any nonGaussian signal in the temperature field arises generically due to higher order effects [46-51].

The situation is different in the case of magnetically induced signals. Magnetic stresses depend quadratically on the field. Hence, even if the fields are assumed to be Gaussian, the corresponding stresses are not. This implies that the 
CMB temperature anisotropy, which these stresses induce, do not have Gaussian statistics. Unlike the case of CMB nonGaussianity from inflationary perturbations, in the case of magnetic fields, the CMB non-Gaussianity is a leading order effect. Thus the study of CMB non-Gaussianity has a special significance in the context of probing and detecting primordial cosmological magnetic fields.

Preliminary studies of such non-Gaussian signals in the CMB induced by primordial magnetic fields have begun [5254], based on earlier calculations of non-Gaussianity in the magnetic stress energy [55]. These have been limited to the scalar mode signals on large scales and further restricted to a component referred to as the compensated scalar mode by [40]. However, the work of Shaw and Lewis [40] has revealed that much larger CMB anisotropies can result from scalar perturbations sourced by the magnetic anisotropic stresses prior to neutrino decoupling (also see $[56-58]$ ). This mode is referred to as the passive scalar mode [40]. In this paper we calculate the contribution to the $\mathrm{CMB}$ bispectrum from the passive scalar mode. As we show here, this contribution greatly dominates over the contribution calculated earlier by two of us [52] (hereafter referred to as SS09), and also calculated by [53]. The current work allows tighter limits to be placed on primordial magnetic fields.

In the next section we describe the properties of the primordial stochastic magnetic field and in section 3 we describe the perturbation induced due to the passive scalar mode. The magnetic CMB temperature anisotropy is described in Section 4 and in its subsections we calculate the three-point correlation of the scalar anisotropic stress in two different configurations. In section 5 we calculate the passive mode reduced bispectrum for the different configurations and in section 6 we use the reduced magnetic bispectra to put constraints on the primordial magnetic field. Our conclusions are summarized in section 7 .

\section{PRIMORDIAL STOCHASTIC MAGNETIC FIELD}

We consider a stochastic magnetic field $B$ which is a Gaussian random field characterized and completely specified by its energy power spectrum $M(k)$. In addition we assume the magnetic field is non-helical.

On galactic and larger scales, any velocity induced by Lorentz forces is generally so small that it does not lead to appreciable distortion of the initial field [59, 60]. Hence, the magnetic field simply redshifts away as $\mathbf{B}(\mathbf{x}, t)=\mathbf{b}_{0}(\mathbf{x}) / a^{2}$, where, $\mathbf{b}_{0}$ is the magnetic field at the present epoch (i.e. at $z=0$ or $a=1$ ).

We define $\mathbf{b}(\mathbf{k})$ as the Fourier transform of the magnetic field $\mathbf{b}_{0}(\mathbf{x})$. The energy power spectrum is defined by the relation $\left\langle b_{i}(\mathbf{k}) b_{j}^{*}(\mathbf{q})\right\rangle=(2 \pi)^{3} \delta(\mathbf{k}-\mathbf{q}) P_{i j}(\mathbf{k}) M(k)$, where $P_{i j}(\mathbf{k})=\left(\delta_{i j}-k_{i} k_{j} / k^{2}\right)$ is the projection operator ensuring $\nabla \cdot \mathbf{b}_{\mathbf{0}}=0$. This gives $\left\langle\mathbf{b}_{0}^{2}\right\rangle=2 \int(d k / k) \Delta_{b}^{2}(k)$, where $\Delta_{b}^{2}(k)=k^{3} M(k) /\left(2 \pi^{2}\right)$ is the power per logarithmic interval in $k$-space residing in the stochastic magnetic field.

As in [61], we assume a power-law magnetic energy spectra, $M(k)=A k^{n}$ that has a cutoff at $k=k_{c}$, where $k_{c}$ is the
Alfvén-wave damping length-scale [59, 60]. We fix $A$ by demanding that the variance of the magnetic field smoothed over a 'galactic' scale, $k_{G}=1 h \mathrm{Mpc}^{-1}$, (using a sharp $k$-space filter) is $B_{0}$. This gives, (for $n>-3$ and for $k<k_{c}$ )

$$
\Delta_{b}^{2}(k)=\frac{k^{3} M(k)}{2 \pi^{2}}=\frac{B_{0}^{2}}{2}(n+3)\left(\frac{k}{k_{G}}\right)^{3+n} .
$$

The magnetic spectral index is restricted to $-3 \lesssim n<$ $-3 / 2$. Blue spectral indices are strongly disfavoured by many observations like the CMB power spectra [28], [29] and especially by the gravitational wave limits of [62].

\section{PASSIVE SCALAR MODE}

The stress tensor (space-space part of the energymomentum tensor) for magnetic fields in terms of the present day magnetic field value $\mathbf{b}_{\mathbf{0}}$ is

$$
T_{j}^{i}(\mathbf{x})=\frac{1}{4 \pi a^{4}}\left(\frac{1}{2} b_{0}^{2}(\mathbf{x}) \delta_{j}^{i}-b_{0}^{i}(\mathbf{x}) b_{0 j}(\mathbf{x})\right)
$$

In Fourier space, the product of magnetic fields becomes a convolution

$$
\begin{gathered}
S_{j}^{i}(\mathbf{k})=\int b^{i}(\mathbf{q}) b_{j}(\mathbf{k}-\mathbf{q}) d^{3} \mathbf{q} \\
T_{j}^{i}(\mathbf{k})=\frac{1}{4 \pi a^{4}}\left(\frac{1}{2} S_{\alpha}^{\alpha}(\mathbf{k}) \delta_{j}^{i}-S_{j}^{i}(\mathbf{k})\right) .
\end{gathered}
$$

This can be expressed in terms of the magnetic perturbations to the energy-momentum tensor as

$$
T_{j}^{i}(\mathbf{k})=p_{\gamma}\left(\Delta_{B} \delta_{j}^{i}+\Pi_{B j}^{i}\right)
$$

where $\Delta_{B}$ and $\Pi_{B}{ }_{j}^{i}$ are the perturbations in the energy density and anisotropic stress, respectively, as defined in [40] and $p_{\gamma}$ is the radiation pressure.

The magnetic stresses are non-linear in the field but we assume that they are always small compared to the total energy density and pressure of the photons, baryons etc. Thus allowing a purely linear treatment of the perturbations. Hence the scalar, vector and tensor perturbations decouple and evolve independently and here we focus on the anisotropic scalar perturbations $\Pi_{B}(\boldsymbol{k})$ which are given by applying the relevant projection operator to $T_{j}^{i}(\mathbf{k})$ [55].

$$
\Pi_{B}(\boldsymbol{k})=-\frac{3}{2}\left(\hat{\boldsymbol{k}}_{i} \hat{\boldsymbol{k}}_{j}-\frac{1}{3} \delta_{i j}\right) \Pi_{B}^{i j}
$$

Note that $\Pi_{B}(\boldsymbol{k})$ of [40] is equal to $-\tau^{S}(\boldsymbol{k})$ of [55].

Such an anisotropic stress term also arises due to neutrinos after they decouple. The net anisotropic stress tensor acts as the source term in the scalar Einstein's equations that lead to the Bardeen equation for the potential. Prior to neutrino decoupling, the only source of anisotropic stress is the magnetic field. Once the neutrinos decouple, the anisotropic stress 
due to neutrinos also contributes but with an opposite sign to that of the magnetic field, thus compensating the contribution from the magnetic field [18]. After compensation there are two modes of perturbation [40]. The first one, called the passive mode is an adiabatic-like mode but has non-Gaussian statistics. It grows logarithmically in amplitude between the epochs of magnetic field generation and neutrino decoupling but then evolves passively after neutrino decoupling. This behaviour has also been confirmed in [57] in the context of deriving the magnetic Sachs-Wolfe effect for a causally generated primordial magnetic field. The second, more well-studied perturbation [23-26], is called the compensated mode.

The final curvature perturbation due to the passive mode is given by equation (86) of Shaw \& Lewis [40],

$$
\zeta=\zeta\left(\tau_{B}\right)-\frac{1}{3} R_{\gamma} \Pi_{B}\left[\ln \left(\frac{\tau_{\nu}}{\tau_{B}}\right)+\left(\frac{5}{8 R_{\nu}}-1\right)\right] .
$$

in the conformal Newtonian gauge. The evolution of the curvature perturbation has also been discussed (in synchronous gauge) in [56] for the case of an extra source of anisotropic stress cancelling the neutrino anisotropic stress. The role of anisotropic stresses on CMB has also been discussed by [63]. Here $R_{\nu} \sim 0.4$ is the fractional contribution of neutrino energy density towards the total energy density of the relativistic component. The fractional contribution of radiation energy density is $R_{\gamma}=1-R_{\nu} \sim 0.6$. The $\zeta\left(\tau_{B}\right)$ term represents all primordial contributions to the curvature perturbation. The $\log$ term shows the growth in the curvature between the epochs of magnetic field generation $\tau_{B}$ and neutrino decoupling $\tau_{\nu}$. This log term is always bigger than 10 for different choices of $\tau_{B}$ so we can neglect the $\left(\left(5 / 8 R_{\nu}\right)-1\right)$ term with less than $5 \%$ error. After neutrino decoupling, the anisotropic stress $\Pi_{B}$ is compensated and there is a remnant adiabatic mode with amplitude given by [40].

$$
\zeta \sim-\frac{1}{3} R_{\gamma} \Pi_{B} \ln \left(\frac{\tau_{\nu}}{\tau_{B}}\right) .
$$

This so-called passive mode evolves passively like an adiabatic perturbation but the statistics of $\Pi_{B}$ are non-Gaussian unlike the standard inflationary adiabatic mode. Also, unlike the compensated mode, the passive mode amplitude depends logarithmically on the epoch of magnetic field generation. Therefore, limits on the primordial magnetic field strength arising from this passive mode will be somewhat sensitive to the assumed model and epoch of magnetic field generation.

\section{MAGNETIC CMB ANISOTROPY AND THREE-POINT CORRELATION}

We consider the $\mathrm{CMB}$ temperature anisotropies that are sourced by the scalar passive mode. On large angular scales the important contribution is via the magnetically induced Sachs-Wolfe effect

$$
\frac{\Delta T}{T}(\boldsymbol{n})=\frac{1}{3} \Phi\left(\boldsymbol{x}_{0}-\boldsymbol{n} D^{*}\right)=\frac{1}{5} \zeta\left(\boldsymbol{x}_{0}-\boldsymbol{n} D^{*}\right)
$$

where the second equality is from, for example, Eq. (10.42) of [64]. The unit vector $\mathbf{n}$ gives the direction of observation and $D^{*}$ is the (angular diameter) distance to the surface of last scatter. Then employing Eq. (8) we get

$$
\begin{aligned}
\frac{\Delta T}{T}(\boldsymbol{n}) & =-\frac{1}{15} R_{\gamma} \ln \left(\frac{\tau_{\nu}}{\tau_{B}}\right) \Pi_{B}\left(\boldsymbol{x}_{0}-\boldsymbol{n} D^{*}\right) \\
& =\mathcal{R}_{p} \Pi_{B}\left(\boldsymbol{x}_{0}-\boldsymbol{n} D^{*}\right) .
\end{aligned}
$$

where we define

$$
\mathcal{R}_{p}=-\frac{1}{15} R_{\gamma} \ln \left(\frac{\tau_{\nu}}{\tau_{B}}\right) .
$$

In SS09 we had calculated the bispectrum due to the compensated scalar mode for which $\Delta T / T=\left(R_{\gamma} / 20\right) \Delta_{B}$ [65]. Equation (10) for the passive scalar mode leads to a $\Delta T / T$ which is larger by a factor $\mathcal{A}=(4 / 3) \ln \left(\tau_{\nu} / \tau_{B}\right)\left(\Pi_{B} / \Delta_{B}\right)$. Assuming that the fractional perturbations $\Delta_{B}$ and $\Pi_{B}$ are of the same order, we get $\mathcal{A}$ of order 50 for $\tau_{B} \approx 10^{14} \mathrm{GeV}$. As we will see below for the magnetic spectra we consider, this alone leads to an enhanced passive scalar mode contribution to the bispectrum of order $\mathcal{A}^{3} \approx 10^{5}$ and thus stronger limits on the primordial magnetic field by a factor $\mathcal{A}^{0.5}$. We assume instantaneous recombination which is a good approximation for large angular scales. There could also be additional integrated Sachs-Wolfe (ISW) contributions to $\Delta T / T(\boldsymbol{n})$.

The CMB temperature fluctuation in a direction $(\boldsymbol{n})$ at the observer can be expanded in terms of the spherical harmonics to give

$$
\frac{\Delta T(\boldsymbol{n})}{T}=\sum_{l m} a_{l m} Y_{l m}(\boldsymbol{n})
$$

where, for the passive scalar mode,

$$
a_{l m}=4 \pi(-i)^{l} \int \frac{d^{3} k}{(2 \pi)^{3}} \mathcal{R}_{p} \Pi_{B}(\boldsymbol{k}) j_{l}\left(k D^{*}\right) Y_{l m}^{*}(\hat{\boldsymbol{k}}) .
$$

Here $\Pi_{B}(\boldsymbol{k})$ is the Fourier transform of $\Pi_{B}(\mathbf{x})$.

The non-Gaussianity in the CMB temperature anisotropy can be evaluated by calculating its 3-point correlation function (in harmonic space), called the bispectrum, $B_{l_{1} l_{2} l_{3}}^{m_{1} m_{2} m_{3}}$. We assume that the magnetic perturbations are uncorrelated with the primary (inflationary) perturbations in the CMB.

In terms of the $a_{l m}$ 's the CMB bispectrum is given by,

$$
B_{l_{1} l_{2} l_{3}}^{m_{1} m_{2} m_{3}}=\left\langle a_{l_{1} m_{1}} a_{l_{2} m_{2}} a_{l_{3} m_{3}}\right\rangle .
$$

From Eq. (13) we can express $B_{l_{1} l_{2} l_{3}}^{m_{1} m_{2} m_{3}}$ as

$B_{l_{1} l_{2} l_{3}}^{m_{1} m_{2} m_{3}}=\mathcal{R}_{p}^{3} \int\left[\prod_{i=1}^{3}(-i)^{l_{i}} \frac{d^{3} k_{i}}{2 \pi^{2}} j_{l_{i}}\left(k_{i} D^{*}\right) Y_{l_{i} m_{i}}^{*}\left(\hat{\boldsymbol{k}}_{i}\right)\right] \zeta_{123}$

with $\zeta_{123}$ defined as,

$$
\zeta_{123}=\left\langle\hat{\Pi}_{B}\left(\boldsymbol{k}_{1}\right) \hat{\Pi}_{B}\left(\boldsymbol{k}_{2}\right) \hat{\Pi}_{B}\left(\boldsymbol{k}_{3}\right)\right\rangle
$$

The function $\zeta_{123}$ is the three-point correlation of $\hat{\Pi}_{B}(\boldsymbol{k})$ and involves a 6 -point correlation function of the magnetic fields. 
Using Wick's theorem it can be written as a function of magnetic spectra in an analogous manner to [55] and Eq. (7) of SS09, $\zeta_{123}=\delta\left(\boldsymbol{k}_{1}+\boldsymbol{k}_{2}+\boldsymbol{k}_{3}\right) \psi_{123}$, where

$$
\begin{gathered}
\psi_{123}=\frac{1}{\left(4 \pi p_{\gamma}\right)^{3}} \int d^{3} s M\left(\left|\boldsymbol{k}_{1}+\boldsymbol{s}\right|\right) M(s) M\left(\left|\boldsymbol{s}-\boldsymbol{k}_{3}\right|\right) \\
\times\left(\mathcal{F}_{\left.\Pi_{B} \Pi_{B} \Pi_{B}\right) .}\right.
\end{gathered}
$$

where $\mathcal{F}_{\Pi_{B} \Pi_{B} \Pi_{B}}$ is the angular component of the three-point correlation $\zeta_{123}$ of the scalar anisotropic stress $\Pi_{B}(\boldsymbol{k})$ in mode-coupling integral $\psi_{123}$.

\section{A. Three-Point Correlation of Scalar Anisotropic Stress}

This angular component $\mathcal{F}_{\Pi_{B} \Pi_{B} \Pi_{B}}$ is given by a 58 term expression in [55] derived by applying relevant projection operators to extract the scalar part of the full bispectrum i.e the six-point correlation of the magnetic fields. In this particular case, the operator is given by $\mathcal{A}_{i j k l m n}=$ $(-1)^{3} \mathcal{Q}_{i j}\left(\boldsymbol{k}_{3}\right) \mathcal{Q}_{k l}\left(\boldsymbol{k}_{1}\right) \mathcal{Q}_{m n}\left(\boldsymbol{k}_{2}\right)$ where $\mathcal{Q}_{a b}(\boldsymbol{k}) \equiv \delta_{a b}-$ $(3 / 2) P_{a b}(\boldsymbol{k})$ and $P_{a b}(\boldsymbol{k})=\delta_{a b}-\hat{\boldsymbol{k}}_{a} \hat{\boldsymbol{k}}_{b}$ is the projection operator. We present the full angular component (in our notation we have absorbed a factor of 8 multiplying $\mathcal{F}$ in [55] into our definition of $\mathcal{F}_{\Pi_{B} \Pi_{B} \Pi_{B}}$ ):

$$
\mathcal{F}_{\Pi_{B} \Pi_{B} \Pi_{B}}=\sum_{n=0}^{6} \mathcal{F}_{\Pi_{B} \Pi_{B} \Pi_{B}}^{n}
$$

with

$$
\begin{aligned}
& \mathcal{F}_{\Pi_{B} \Pi_{B} \Pi_{B}}^{0}=-9 \\
& \mathcal{F}_{\Pi_{B} \Pi_{B} \Pi_{B}}^{1}=0 \\
& \mathcal{F}_{\Pi_{B} \Pi_{B} \Pi_{B}}^{2}=\left(\bar{\beta}^{2}+\bar{\gamma}^{2}+\bar{\mu}^{2}+9\left(\theta_{13}^{2}+\theta_{23}^{2}+\theta_{12}^{2}\right)+3\left(\alpha_{3}^{2}+\alpha_{1}^{2}+\alpha_{2}^{2}+\beta_{3}^{2}+\beta_{1}^{2}+\beta_{2}^{2}+\gamma_{3}^{2}+\gamma_{1}^{2}+\gamma_{2}^{2}\right)\right) \\
& \mathcal{F}_{\Pi_{B} \Pi_{B} \Pi_{B}}^{3}=-3\left(\bar{\mu}\left(\beta_{3} \gamma_{3}+\beta_{1} \gamma_{1}+\beta_{2} \gamma_{2}+\frac{1}{3} \bar{\beta} \bar{\gamma}\right)+\bar{\gamma}\left(\alpha_{3} \gamma_{3}+\alpha_{1} \gamma_{1}+\alpha_{2} \gamma_{2}\right)+\bar{\beta}\left(\alpha_{3} \beta_{3}+\alpha_{1} \beta_{1}+\alpha_{2} \beta_{2}\right)\right. \\
& +3 \theta_{13}\left(\alpha_{3} \alpha_{1}+\beta_{3} \beta_{1}+\gamma_{3} \gamma_{1}\right)+3 \theta_{23}\left(\alpha_{3} \alpha_{2}+\beta_{3} \beta_{2}+\gamma_{3} \gamma_{2}\right)+3 \theta_{12}\left(\alpha_{1} \alpha_{2}+\beta_{1} \beta_{2}+\gamma_{1} \gamma_{2}\right) \\
& \left.+9 \theta_{13} \theta_{23} \theta_{12}\right) \\
& \mathcal{F}_{\Pi_{B} \Pi_{B} \Pi_{B}}^{4}=3\left(\bar{\gamma} \bar{\mu} \alpha_{3} \beta_{3}+\bar{\beta} \bar{\mu} \alpha_{1} \gamma_{1}+\bar{\beta} \bar{\gamma} \beta_{2} \gamma_{2}+3\left(\bar{\mu} \theta_{13} \beta_{3} \gamma_{1}+\bar{\gamma} \theta_{23} \alpha_{3} \gamma_{2}+\bar{\beta} \theta_{12} \alpha_{1} \beta_{2}\right)\right. \\
& +3\left(\alpha_{3} \beta_{3}\left(\alpha_{1} \beta_{1}+\alpha_{2} \beta_{2}\right)+\alpha_{1} \gamma_{1}\left(\alpha_{3} \gamma_{3}+\alpha_{2} \gamma_{2}\right)+\beta_{2} \gamma_{2}\left(\beta_{3} \gamma_{3}+\beta_{1} \gamma_{1}\right)\right) \\
& \left.+9\left(\theta_{13} \theta_{23} \gamma_{1} \gamma_{2}+\theta_{13} \theta_{12} \beta_{3} \beta_{2}+\theta_{23} \theta_{12} \alpha_{3} \alpha_{1}\right)\right) \\
& \mathcal{F}_{\Pi_{B} \Pi_{B} \Pi_{B}}^{5}=-9\left(\bar{\mu} \alpha_{3} \beta_{3} \alpha_{1} \gamma_{1}+\bar{\gamma} \alpha_{3} \beta_{3} \beta_{2} \gamma_{2}+\bar{\beta} \alpha_{1} \gamma_{1} \beta_{2} \gamma_{2}+3\left(\theta_{13} \beta_{3} \gamma_{1} \beta_{2} \gamma_{2}+\theta_{23} \alpha_{3} \alpha_{1} \gamma_{1} \gamma_{2}+\theta_{12} \alpha_{3} \beta_{3} \alpha_{1} \beta_{2}\right)\right) \\
& \mathcal{F}_{\Pi_{B} \Pi_{B} \Pi_{B}}^{6}=27 \alpha_{3} \beta_{3} \alpha_{1} \gamma_{1} \beta_{2} \gamma_{2} .
\end{aligned}
$$

where

$$
\bar{\beta}=\left(\widehat{\boldsymbol{s}} \cdot \widehat{\boldsymbol{k}_{3}-\boldsymbol{s}}\right), \bar{\gamma}=\left(\widehat{\boldsymbol{s}} \cdot \widehat{\boldsymbol{k}_{1}+\boldsymbol{s}}\right), \bar{\mu}=\left(\widehat{\boldsymbol{k}_{3}-\boldsymbol{s}} \cdot \widehat{\boldsymbol{k}_{1}+\boldsymbol{s}}\right) .
$$

where the hat on a vector denotes its unit vector.

Also,

$$
\alpha_{a}=\hat{\boldsymbol{k}}_{a} \cdot \boldsymbol{s}, \quad \beta_{a}=\hat{\boldsymbol{k}}_{a} \cdot \widehat{\boldsymbol{k}_{3}-\boldsymbol{s}}, \quad \gamma_{a}=\hat{\boldsymbol{k}}_{a} \cdot \widehat{\boldsymbol{k}_{1}+\boldsymbol{s}}
$$

and

$$
\theta_{a b}=\hat{\boldsymbol{k}}_{a} \cdot \hat{\boldsymbol{k}}_{b}
$$

where our angle definitions are consistent with [55] and slightly different to SS09.
For simplicity, we evaluate this $\mathcal{F}_{\Pi_{B} \Pi_{B} \Pi_{B}}$ expression to find the bispectrum in the following two cases below. We expect that the magnitude of the bispectrum will be of similar order for a general case.

Case I - Consider any bispectrum configuration but include only the $s$-independent terms (constant for any particular configuration) in the $\mathcal{F}_{\Pi_{B} \Pi_{B} \Pi_{B}}$ expression. Then the $s$-integral in Eq. (17) is performed without reference to any particular bispectrum configuration.

Case II - The squeezed collinear configuration where we calculate fully the mode-coupling integral over all angular terms in the $\mathcal{F}_{\Pi_{B} \Pi_{B} \Pi_{B}}$ expression. 
TABLE I. The sum of $s$-independent terms $m=\mathcal{F}_{\Pi_{B}}^{\mathrm{I}} \Pi_{B} \Pi_{B}$ in four different configurations $\left(\boldsymbol{k}_{1}, \boldsymbol{k}_{2}, \boldsymbol{k}_{3}\right)$ for evaluating the Case I bispectrum.

\begin{tabular}{|c|c|c|c|}
\hline Configuration & $\left(\boldsymbol{k}_{1}, \boldsymbol{k}_{2}, \boldsymbol{k}_{3}\right)$ & $\left(\theta_{12}, \theta_{23}, \theta_{13}\right)$ & $m$ \\
\hline $\begin{array}{c}\text { local } \\
\text { isosceles }\end{array}$ & $\begin{array}{c}k_{1} \sim k_{3} \\
k_{2} \ll k_{1}, k_{3}\end{array}$ & $(0,0,-1)$ & 0 \\
\hline equilateral & $k_{1} \sim k_{2} \sim k_{3}$ & $\left(\frac{1}{2}, \frac{1}{2}, \frac{1}{2}\right)$ & -5.625 \\
\hline $\begin{array}{l}\text { squeezed } \\
\text { collinear }\end{array}$ & $\begin{array}{c}k_{1} \sim k_{3} \\
k_{2} \ll k_{1}, k_{3} \\
\hat{\boldsymbol{k}}_{1}=\hat{\boldsymbol{k}}_{2}=-\hat{\boldsymbol{k}}_{3}\end{array}$ & $(1,-1,-1)$ & $-8^{\mathrm{a}}$ \\
\hline $\begin{array}{l}\text { midpoint } \\
\text { collinear }\end{array}$ & $\begin{array}{c}k_{1} \sim k_{2} \sim \frac{k_{3}}{2} \\
\hat{\boldsymbol{k}}_{1}=\hat{\boldsymbol{k}}_{2}=-\hat{\boldsymbol{k}}_{3}\end{array}$ & $(1,-1,-1)$ & -9 \\
\hline
\end{tabular}

${ }^{\text {a }}$ For the squeezed collinear configuration case, $\mathcal{F}_{\Pi_{B} \Pi_{B} \Pi_{B}}$ picks up another term $\bar{\mu}^{2} \sim 1$

\section{B. Case I: Evaluation with $s$-independent Terms in $\mathcal{F}_{\Pi_{B} \Pi_{B} \Pi_{B}}$}

The $s$-independent terms include $\theta_{12}, \theta_{23}, \theta_{13}$ that are constant for a given $\left(\boldsymbol{k}_{1}, \boldsymbol{k}_{2}, \boldsymbol{k}_{3}\right)$ configuration. For each configuration considered, we calculate the sum of the five $s$ independent terms in Eq. (19) for $\mathcal{F}_{\Pi_{B} \Pi_{B} \Pi_{B}}$

$$
\mathcal{F}_{\Pi_{B} \Pi_{B} \Pi_{B}}^{\mathrm{I}}=-9+9\left(\theta_{12}^{2}+\theta_{23}^{2}+\theta_{13}^{2}\right)-27\left(\theta_{13} \theta_{12} \theta_{23}\right) .
$$

We give in Table I the values of $m=\mathcal{F}_{\Pi_{B} \Pi_{B} \Pi_{B}}\left(\boldsymbol{k}_{1}, \boldsymbol{k}_{2}, \boldsymbol{k}_{3}\right)$ for specific configurations $\left(\boldsymbol{k}_{1}, \boldsymbol{k}_{2}, \boldsymbol{k}_{3}\right)$. Note that $m$ happens to vanish exactly for the local isosceles configuration. Hence the Case I mode-coupling integral reduces to

$$
\psi_{123}=\frac{m}{\left(4 \pi p_{\gamma}\right)^{3}} \mathcal{I}=\frac{3^{3} m}{\left(4 \pi \rho_{0}\right)^{3}} \mathcal{I}
$$

where $\rho_{0}$ is the present day energy density of radiation and

$$
\begin{aligned}
\mathcal{I}= & \int d^{3} s M\left(\left|\boldsymbol{k}_{1}+\boldsymbol{s}\right|\right) M(s) M\left(\left|\boldsymbol{s}-\boldsymbol{k}_{3}\right|\right) \\
= & 2 \pi A^{3} \int_{-1}^{1} d \mu \int_{0}^{\infty} d s s^{n+2}\left(s^{2}+k_{1}^{2}+2 s k_{1} \nu\right)^{\frac{n}{2}} \\
& \times\left(s^{2}+k_{3}^{2}-2 s k_{3} \mu\right)^{\frac{n}{2}}
\end{aligned}
$$

where $\quad \nu=\hat{\boldsymbol{k}}_{1} \cdot \hat{\boldsymbol{s}} \quad$ and $\quad \mu=\hat{\boldsymbol{k}}_{3} \cdot \hat{\boldsymbol{s}}$.

We perform the mode-coupling integral using the technique discussed in [16, 17, 61]. As $m$ vanishes for the local isosceles configuration, $\psi_{123}$ is zero for this configuration. For the equilateral and squeezed collinear configurations we split the $s$ integral into two sub-ranges $0<s<k_{1} \sim k_{3}$ and $s>k_{1} \sim k_{3}$ We then approximate the integrands by assuming $s \ll k_{1} \sim k_{3}$ and $s \gg k_{1} \sim k_{3}$ for the respective subranges. For the midpoint collinear configuration we split the $s$ integral into two sub-ranges $0<s<k_{1}$ and $s>2 k_{1} \sim k_{3}$ while neglecting the very small contribution from the middle sub-range $k_{1}<s<2 k_{1} \sim k_{3}$. Again, we approximate the integrands by assuming $s \ll k_{1}$ and $s \gg 2 k_{1} \sim k_{3}$ for the respective sub-ranges. To derive numerical estimates of the bispectrum and magnetic field strengths we will focus on nearly scale-invariant spectra (which can be produced by an acausal mechanism like inflation), i.e $n \rightarrow-3$, which yield

$$
\begin{aligned}
\mathcal{I} & \simeq 4 \pi A^{3}\left[\frac{k_{1}^{2 n+3} k_{3}^{n}}{n+3}-\frac{k_{3}^{3 n+3}}{3 n+3}\right] \\
& \simeq 4 \pi A^{3} \frac{k_{1}^{2 n+3} k_{3}^{n}}{n+3}
\end{aligned}
$$

The latter equation is obtained in the limit $n \rightarrow-3$ where we can neglect the terms with $(3 n+3)^{-1}$ compared to $(n+3)^{-1}$. Hence the mode-coupling integral for Case I - taking only $s$ independent angular part - is

$$
\psi_{123}=(4)^{4} m \frac{\pi^{7}}{k_{G}^{6}}(n+3)^{2}\left(\frac{k_{1}}{k_{G}}\right)^{2 n+3}\left(\frac{k_{3}}{k_{G}}\right)^{n} V_{A}^{6} .
$$

Here we have defined $V_{A}$, the Alfvén velocity in the radiation dominated era as [60]

$$
V_{A}=\frac{B_{0}}{\left(16 \pi \rho_{0} / 3\right)^{1 / 2}} \approx 3.8 \times 10^{-4} B_{-9},
$$

with $B_{-9} \equiv\left(B_{0} / 10^{-9}\right.$ Gauss $)$.

\section{Case II: Squeezed Collinear Configuration - All Angular Terms}

For Case II - We take the squeezed collinear bispectrum configuration as $k_{1} \sim k_{3}$ and $k_{2} \ll k_{1}, k_{3}$ and $\hat{\boldsymbol{k}}_{1}=\hat{\boldsymbol{k}}_{2}=$ $-\hat{\boldsymbol{k}}_{3}$. One wavevector $\left(\boldsymbol{k}_{2}\right)$ is negligibly small compared to the other two which are also in exactly opposite directions and of a similar magnitude. This affords considerable simplification and reduction of the angular terms given by Eq. (19) in doing the mode-coupling integral. Using $\boldsymbol{k}_{3} \approx-\boldsymbol{k}_{1}$ we see that

$$
\begin{gathered}
\bar{\beta}=\left(\widehat{\boldsymbol{s}} \cdot \widehat{\boldsymbol{k}_{3}-\boldsymbol{s}}\right) \approx\left(\widehat{\boldsymbol{s}} \cdot \widehat{-\boldsymbol{k}_{1}-\boldsymbol{s}}\right) \approx-\left(\widehat{\boldsymbol{s}} \cdot \widehat{\boldsymbol{k}_{1}+\boldsymbol{s}}\right) \approx-\bar{\gamma} \\
\bar{\mu}=\left(\widehat{\boldsymbol{k}_{3}-\boldsymbol{s}} \cdot \widehat{\boldsymbol{k}_{1}+\boldsymbol{s}}\right) \approx\left(\widehat{\boldsymbol{k}_{1}-\boldsymbol{s}} \cdot \widehat{\boldsymbol{k}_{1}+\boldsymbol{s}}\right) \\
\approx-\left(\widehat{\boldsymbol{k}_{1}+\boldsymbol{s}} \cdot \widehat{\boldsymbol{k}_{1}+\boldsymbol{s}}\right) \approx-1 \\
\alpha_{1}=\alpha_{2}=-\alpha_{3}=\alpha=\nu=-\mu \\
\beta_{1}=\beta_{2}=-\beta_{3}=\beta \\
\gamma_{1}=\gamma_{2}=-\gamma_{3}=\gamma \\
\theta_{12}=1, \theta_{23}=\theta_{13}=-1
\end{gathered}
$$


These relations substituted into Eq. (19) reduce the 58 terms to a 10-term angular expression for the squeezed collinear configuration

$$
\begin{aligned}
\mathcal{F}_{\Pi_{B} \Pi_{B} \Pi_{B}}= & -8+\bar{\beta}^{2}+9\left(\mu^{2}+2 \gamma^{2}\right)+6 \mu \bar{\beta} \gamma+3 \bar{\beta}^{2} \gamma^{2} \\
& -9 \gamma^{2}\left(3 \mu^{2}+\gamma^{2}\right)-18 \mu \bar{\beta} \gamma^{3}+27 \mu^{2} \gamma^{4} .
\end{aligned}
$$

We again perform the mode-coupling as discussed above and split the $s$ integral into two sub-ranges $0<s<k_{1} \sim k_{3}$ and $s>k_{1} \sim k_{3}$. Once again we approximate the integrands by assuming $s \ll k_{1} \sim k_{3}$ and $s \gg k_{1} \sim k_{3}$ for the respective sub-ranges to give

$$
\psi_{123}=\frac{1}{\left(4 \pi p_{\gamma}\right)^{3}} \mathcal{I}=\left(\frac{3}{4 \pi \rho_{0}}\right)^{3} \mathcal{I}
$$

where

$$
\begin{aligned}
\mathcal{I}= & \int d^{3} s M\left(\left|\boldsymbol{k}_{1}+\boldsymbol{s}\right|\right) M(s) M\left(\left|\boldsymbol{s}-\boldsymbol{k}_{3}\right|\right) \\
& \times\left[-8+\bar{\beta}^{2}+9\left(\mu^{2}+2 \gamma^{2}\right)+6 \mu \bar{\beta} \gamma+3 \bar{\beta}^{2} \gamma^{2}\right. \\
& \left.-9 \gamma^{2}\left(3 \mu^{2}+\gamma^{2}\right)-18 \mu \bar{\beta} \gamma^{3}+27 \mu^{2} \gamma^{4}\right]
\end{aligned}
$$

Performing the integrals for all the terms, with $n \rightarrow-3$, we find that there is considerable though incomplete cancellation between the 10 terms

$$
\begin{aligned}
\mathcal{I} & \simeq 2 \pi A^{3} \frac{k_{1}^{2 n+3} k_{3}^{n}}{n+3} \times \\
& {\left[-16+\frac{2}{3}+6+36+4+2-18-18-12+18\right] } \\
\simeq & 2 \pi A^{3} \frac{k_{1}^{2 n+3} k_{3}^{n}}{n+3}\left[\frac{8}{3}\right]
\end{aligned}
$$

We draw attention to how the full evaluation gives a result for $\psi_{123}$ that is of opposite sign and one-sixth magnitude (8/3) of the value if we only consider the constant term (16) for this squeezed collinear configuration. The sign of the mode-coupling integral is important as the bispectrum is not a positive-definite quantity (unlike the power spectrum) and the observed limits on the bispectrum may also be asymmetric about zero. Then the Case II - squeezed collinear modecoupling integral is

$$
\psi_{123}=\left[\frac{8}{3}\right] 2(4)^{3} \frac{\pi^{7}}{k_{G}^{6}}(n+3)^{2}\left(\frac{k_{1}}{k_{G}}\right)^{2 n+3}\left(\frac{k_{3}}{k_{G}}\right)^{n} V_{A}^{6} .
$$

As the n-dependence of $\psi_{123}$ is identical for both Case I and Case II we can write

$$
\psi_{123}=\mathcal{K}\left[2(4)^{3} \frac{\pi^{7}}{k_{G}^{6}}(n+3)^{2}\left(\frac{k_{1}}{k_{G}}\right)^{2 n+3}\left(\frac{k_{3}}{k_{G}}\right)^{n} V_{A}^{6} \cdot\right]
$$

where

$$
\mathcal{K}= \begin{cases}2 m & \text { Case I } \\ \frac{8}{3} & \text { Case II }\end{cases}
$$

\section{PASSIVE SCALAR CMB BISPECTRUM}

Using Eq. (40) in Eq. (15) we can evaluate the CMB bispectrum for the passive scalar mode for both Case I and II. The algebraic steps are the same as those for the compensated scalar mode in SS09. We express the delta function present in $\zeta_{123}$ in its integral form $\delta(\boldsymbol{k})=\left(1 /(2 \pi)^{3}\right) \int d^{3} x \exp (i \boldsymbol{k} \cdot \boldsymbol{x})$, use the spherical wave expansion of the exponential terms, substitute it into Eq. (15), and integrate over the angular parts of $\left(\boldsymbol{k}_{1}, \boldsymbol{k}_{2}, \boldsymbol{k}_{3}, \boldsymbol{x}\right)$. This algebra is also very similar to that in the calculation of the primordial bispectrum [49]. Then it becomes possible to write the bispectrum $B_{l_{1} l_{2} l_{3}}^{m_{1} m_{2} m_{3}}$, in terms of a reduced bispectrum $b_{l_{1} l_{2} l_{3}}$ (also referred to as the KomatsuSpergel estimator [47]) as

$$
B_{l_{1} l_{2} l_{3}}^{m_{1} m_{2} m_{3}}=\mathcal{G}_{m_{1} m_{2} m_{3}}^{l_{1} l_{2} l_{3}} b_{l_{1} l_{2} l_{3}}
$$

where

$$
\begin{aligned}
b_{l_{1} l_{2} l_{3}}= & \left(\frac{\mathcal{R}_{p}}{\pi^{2}}\right)^{3} \int x^{2} d x \\
& \times \prod_{i=1}^{3} \int k_{i}^{2} d k_{i} j_{l_{i}}\left(k_{i} x\right) j_{l_{i}}\left(k_{i} D^{*}\right) \psi_{123}
\end{aligned}
$$

and we have introduced the Gaunt integral

$$
\mathcal{G}_{m_{1} m_{2} m_{3}}^{l_{1} l_{2} l_{3}}=\int d \Omega Y_{l_{1} m_{1}} Y_{l_{2} m_{2}} Y_{l_{3} m_{3}} .
$$

We substitute Eq. (40) into Eq. (43) for the reduced bispectrum. The integrals over $k_{2}$ can be immediately done using the spherical Bessel function closure relation

$$
\int k_{2}^{2} d k_{2} j_{l_{2}}\left(k_{2} x\right) j_{l_{2}}\left(k_{2} D^{*}\right)=\left(\pi / 2 x^{2}\right) \delta\left(x-D^{*}\right) .
$$

and the delta function makes the $x$-integral trivial. We are then left with integrals over $k_{1}$ and $k_{3}$ given by

$$
\begin{aligned}
b_{l_{1} l_{2} l_{3}}= & \mathcal{K}\left(\frac{\mathcal{R}_{p}}{\pi^{2}}\right)^{3} \frac{\pi}{2}\left[2(4)^{3} \pi^{7}(n+3)^{2} V_{A}^{6} \cdot\right] \\
& \times\left[\int \frac{d k_{3}}{k_{3}} j_{l_{3}}^{2}\left(k_{3} D^{*}\right)\left(\frac{k_{3}}{k_{G}}\right)^{n+3}\right] \\
& \times\left[\int \frac{d k_{1}}{k_{1}} j_{l_{1}}^{2}\left(k_{1} D^{*}\right)\left(\frac{k_{1}}{k_{G}}\right)^{2(n+3)}\right]
\end{aligned}
$$

The $k_{3}$ and $k_{1}$ integrals are similar in form to the usual SachsWolfe term (for power-law magnetic spectra) and can be evaluated analytically for any n (Eq. 6.574.2 of [66]) in terms of Gamma functions. For $n \rightarrow-3$ we have

$$
\begin{aligned}
b_{l_{1} l_{2} l_{3}}= & \mathcal{K}\left(\frac{\mathcal{R}_{p}}{\pi^{2}}\right)^{3} \frac{\pi}{2}\left[2(4)^{3} \pi^{7}(n+3)^{2} V_{A}^{6} \cdot\right] \\
& \times \frac{1}{2 l_{3}\left(l_{3}+1\right)} \frac{1}{2 l_{1}\left(l_{1}+1\right)}
\end{aligned}
$$

or

$l_{1}\left(l_{1}+1\right) l_{3}\left(l_{3}+1\right) b_{l_{1} l_{2} l_{3}}=\mathcal{K} \mathcal{R}_{p}{ }^{3}\left(\frac{\pi}{2}\right)^{2}(4)^{3}(n+3)^{2} V_{A}^{6}$ 
where a factor of $1 /\left(D^{*} k_{G}\right)^{3(n+3)}$ that also appears has been approximated to unity for the case $n \rightarrow-3$ of a scaleinvariant magnetic field index.

For Case I - The bispectrum considering only constant angular terms

$$
\begin{aligned}
l_{1}\left(l_{1}+1\right) l_{3}\left(l_{3}+1\right) b_{l_{1} l_{2} l_{3}} \approx & \left(-1.35 \times 10^{-16}\right)\left(\frac{3 m}{4}\right) \\
& \times\left(\frac{n+3}{0.2}\right)^{2}\left(\frac{B_{-9}}{3}\right)^{3}
\end{aligned}
$$

where we have used $\tau_{B} \approx 10^{14} \mathrm{GeV}$ corresponding to a possible inflationary epoch for magnetic field generation. This evaluates in different configurations to

$$
\begin{aligned}
& l_{1}\left(l_{1}+1\right) l_{3}\left(l_{3}+1\right) b_{l_{1} l_{2} l_{3}} \approx \\
& \left(10^{-16}\right)\left(\frac{n+3}{0.2}\right)^{2}\left(\frac{B_{-9}}{3}\right)^{3} \times\left\{\begin{array}{ll}
0 & \text { local isosceles } \\
5.7 & \text { equilateral } \\
8.1 & \text { squeezed collinear } \\
9.2 & \text { midpoint collinear }
\end{array}\right\}
\end{aligned}
$$

We see that the constant-term only evaluation gives a bispectrum of order $6-9 \times 10^{-16}$ independent of the exact configuration. The exception is the local isosceles case (where the constant term sum happens to vanish) but if a full evaluation over all angular terms is done, it yields a non-zero bispectrum.

For Case II - The squeezed collinear bispectrum considering all angular terms

$$
\begin{aligned}
l_{1}\left(l_{1}+1\right) l_{3}\left(l_{3}+1\right) b_{l_{1} l_{2} l_{3}} \approx & \left(-1.35 \times 10^{-16}\right) \\
& \times\left(\frac{n+3}{0.2}\right)^{2}\left(\frac{B_{-9}}{3}\right)^{3}
\end{aligned}
$$

The full evaluation of all angular terms for the squeezed collinear case produces two important changes (compared to squeezed collinear in Case I evaluation): the sign of the bispectrum has changed to negative (angular terms in modecoupling contribute with different signs and change the total sign) and it's value had diminished by factor of 6 .

Note that the values of the reduced bispectrum given by Equations (50) and (51) are $\sim 10^{6}$ times larger than the values obtained for the compensated scalar mode in SS09. This is essentially due to the large value of $\mathcal{A}^{3}$ as discussed in section 4.

A numerical evaluation of the mode-coupling integral involves an integrable singularity at $s=\boldsymbol{k}_{3}$ and $s=-\boldsymbol{k}_{1}$. The singularity is integrable (even without a large-scale cutoff) provided we consider the mode-coupling integral over $s$ together with the $k_{1}$ and $k_{3}$ integrals. We defer this numerical investigation for later work and proceed with our analytic results.

\section{CONSTRAINT ON PRIMORDIAL MAGNETIC FIELD}

In order to put constraints on the primordial magnetic field, we compare our magnetic reduced bispectrum with the reduced bispectrum that arises from non-linear terms in the gravitational potential. These have a value given by

$$
l_{1}\left(l_{1}+1\right) l_{3}\left(l_{3}+1\right) b_{l_{1} l_{2} l_{3}} \sim 4 \times 10^{-18} f_{N L}
$$

characterized by $f_{N L}([50])$.

The reduced bispectrum for the magnetic compensated scalar mode [52, 53] was a factor of a few times $10^{4}$ smaller than the standard inflationary signal (for models with $f_{N L} \sim$ 1). However, we find that the magnitude of the corresponding reduced bispectrum for the magnetic passive scalar mode, calculated above, is $\sim 30$ times larger (Case II) or even up to $\sim 200$ times larger (Case I) than the inflationary signal. Both these magnetic bispectra values are for field strength of $3 \mathrm{nG}$ and $n=-2.8$, close to scale-invariant spectrum.

Conversely, we can put upper limits on the primordial magnetic field by equating the magnetic bispectrum to the observed upper limit for the inflationary bispectrum. This is evaluated using the latest WMAP7 limits (95\% CL) on nonGaussianity in the observed CMB [67], $-10<f_{N L}^{l o c}<74$, taking the appropriate side of these limits for the different signs of bispectrum. Since we have considered only the Sachs-Wolfe contribution to magnetic field-induced temperature anisotropies, our magnetic bispectra will be accurate only on large scales. However, the $f_{N L}$ we are using for comparison to data comes from WMAP data that has a maximum multipole of $l \sim 750$ and therefore the bispectra comparison is not exact. Yet our $B_{0}$ limits are expected to be fairly robust as $B_{0}$ depends very weakly $\left(B_{0} \propto f_{N L}^{1 / 6}\right.$ ) on $f_{N L}$ as also discussed in SS09. We obtain the following upper limits for the magnetic field in different cases:

Case I: For any bispectrum configuration considering only $s$-independent terms

$$
B_{0}<3 \mathrm{nG}
$$

For individual configurations the magnetic field limits are: equilateral $(2.7 \mathrm{nG})$, squeezed collinear $(2.5 \mathrm{nG})$ and midpoint collinear $(2.5 \mathrm{nG})$, all for $\tau_{B} \approx 10^{14} \mathrm{GeV}$ corresponding to the inflationary epoch.

Case II: For the squeezed collinear bispectrum configuration, including all angular terms, we obtain an upper limit

$$
B_{0}<2 \mathrm{nG}
$$

where this magnetic field limit value $(2.4 \mathrm{nG})$ is derived using $\tau_{B} \approx 10^{14} \mathrm{GeV}$ and $-10<f_{N L}^{l o c}$.

It is currently unclear what would be an appropriate epoch to adopt for the generation of a large-scale primordial cosmic magnetic field [31, 35-39]. We recall that via the logarithmic factor in Eq. (8), there is a weak dependence of $B_{0}$ on $\tau_{B}$, the magnetic field generation epoch. We find a $2-4 \mathrm{nG}$ variation in the $B_{0}$ upper limit when $\tau_{B}$ is varied between the inflationary and electroweak epochs $\tau_{B} \approx 10^{14} \rightarrow 10^{3} \mathrm{GeV}$, taken as the earliest and latest possible epochs of magnetic field generation. However, as the electroweak transition is not expected to give a scale-invariant magnetic field spectrum, we caution that the choice of electroweak epoch may be too late to generate a field with $n \rightarrow-3$. Hence, the variation in the magnetic field upper limit quoted above may be an overestimate. 


\section{CONCLUSION}

We have calculated here the CMB bispectrum, on large angular scales, due to the passive scalar mode. This mode is sourced by the magnetic scalar anisotropic stress before neutrino decoupling. The CMB bispectrum due to the passive scalar mode is more than two orders of magnitude larger than the bispectrum due to the primary inflation-induced scalar mode, for $f_{N L} \sim 1$ and $B_{0} \sim 3 \mathrm{nG}$. It is also a factor of $10^{6}$ times greater than that previously calculated for the compensated scalar mode [52, 53]. This is the first calculation of a type of magnetic contribution to $\mathrm{CMB}$ bispectrum that can clearly dominate over the primary bispectrum at large scales. In the CMB power spectrum, by contrast, the passive scalar mode signal is highly sub-dominant to the inflationary signal [40]. Our work thus shows that the magnetically induced signals, being intrinsically non-Gaussian, could be more easily distinguished from the primary $\mathrm{CMB}$ anisotropies, when one considers the bispectrum rather than the power spectrum.

Using the WMAP7 limits for $f_{N L}$ we have placed an upper limit on the strength of the primordial magnetic field $B_{0}<2$ $\mathrm{nG}$ which is more than an order of magnitude stronger than the limit from the compensated scalar mode $(35 \mathrm{nG})$ obtained in SS09. The passive mode limit is only weakly (logarithmically) dependent on the epoch of magnetic field generation $\tau_{B}$. We note that for scale-invariant magnetic spectra $n \rightarrow-3$, the bispectrum and the derived magnetic field limit are independent of both the assumed cosmological model as well as our choice of smoothing scale $k_{G}$ for the stochastic magnetic field $B_{0}$.

The primordial magnetic field limit is expected to improve if the ISW effect and full scalar anisotropies are taken into account. We also expect stronger constraints to follow from the tensor and vector mode bispectra that dominate at low and high $l$, respectively. Better observational limits on $\mathrm{CMB}$ non-Gaussianity from PLANCK data are expected to further tighten primordial magnetic field limits.

\section{ACKNOWLEDGEMENTS}

PT would like to acknowledge the assistance from Sri Venkateswara College, University of Delhi, in pursuing this work. PT and TRS would like to acknowledge the IUCAA Associateship Program as well as the facilities at the IUCAA Resource Center, University of Delhi.
[1] R. Beck, Astrophys. Space Sci. Trans. 5, 43 (2009).

[2] C. Vogt and T. A. Enßlin, Astron. \& Astrophys. 412, 373 (2003).

[3] A. Brandenburg and K. Subramanian, Phys. Rep. 417, 1 (2005).

[4] R. M. Kulsrud and E. G. Zweibel, Rep. Prog. Phys. 71, 4, 046901 (2008).

[5] R. Beck, A. Brandenburg, D. Moss, A. Shukurov and D. Sokoloff, Annu. Rev. Astron. Astrophys. 34, 155 (1996).

[6] M. L. Bernet, F. Miniati, S. J. Lilly, P. P. Kronberg and M. Dessauges-Zavadsky, Nature 454, 7202, 302 (2008).

[7] P. P. Kronberg, M. L. Bernet, F. Miniati, S. J. Lilly, M. B. Short, D. M. Higdon, Astrophys. J. 676, 70 (2008).

[8] K.-T. Kim, P. P. Kronberg, G. Giovannini and T. Venturi, Nature 341, 720 (1989).

[9] A. Ando and A Kusenko, ArXiv e-prints (2010), arXiv:1005.1924v2 [astro-ph.HE].

[10] A. Neronov and I Vovk, Science 328, 73 (2010).

[11] K. Subramanian, Astron. Nach. 327, 333 (2006).

[12] R. Durrer, New Astron. Rev. 51, 275 (2007).

[13] J. D. Barrow, P. G. Ferreira and J. Silk, Phys. Rev. Lett. 78, 3610 (1997).

[14] K. Subramanian and J. D. Barrow, Phys. Rev. Lett. 81, 3575 (1998).

[15] R. Durrer, P. G. Ferreira, T. Kahniashvili, Phys. Rev. D 61, 043001 (2000).

[16] T. R. Seshadri and K. Subramanian, Phys. Rev. Lett. 87, 101301 (2001).

[17] A. Mack, T. Kahniashvili and A. Kosowsky, Phys. Rev. D 65, 123004 (2002).

[18] A. Lewis, Phys. Rev. D 70, 043011 (2004).

[19] T. R. Seshadri and K. Subramanian, Phys. Rev. D 72, 023004 (2005).

[20] R. Gopal and S. K. Sethi, Phys. Rev. D 72, 103003 (2005).
[21] T. Kahniashvili and B. Ratra, Phys. Rev. D 71, 103006 (2005).

[22] M. Giovannini, Phys. Rev. D 74, 063002 (2006).

[23] M. Giovannini and K. E. Kunze, Phys. Rev. D 77, 063003 (2008).

[24] D. G. Yamazaki, K. Ichiki, T. Kajino, and G. J. Mathews, Phys. Rev. D 77, 043005 (2008).

[25] F. Finelli, F. Paci and D. Paoletti, Phys. Rev. D 78, 023510 (2008).

[26] D. Paoletti, F. Finelli and F. Paci, Mon. Not. R. Astron. Soc. 396, 1, 523 (2008).

[27] M. Giovannini, Phys. Rev. D 79, 121302 (2009).

[28] D. G. Yamazaki, K. Ichiki, T. Kajino and G. Mathews, Phys. Rev. D 81, 023008 (2010).

[29] D. Paoletti and F. Finelli, ArXiv e-prints (2010), arXiv:1005.0148 [astro-ph.CO].

[30] J. R. Shaw and A. Lewis, ArXiv e-prints (2010), arXiv:1006.4242 1 [astro-ph.CO].

[31] L. M. Widrow, Rev. Mod. Phys. 74, 775 (2002).

[32] H. K. Moffatt, Magnetic Field Generation in Electrically Conducting Fluids (Cambridge University Press, Cambridge, 1978).

[33] F. Krause, K.-H. Rädler, Mean-field Magnetohydrodynamics and Dynamo Theory (Pergamon Press, Oxford, 1980).

[34] A. A. Ruzmaikin, A. Shukurov and D. Sokoloff, Magnetic Fields of Galaxies (Kluwer, Dordrecht, 1988).

[35] M. Turner, L. M. Widrow, Phys. Rev. D 37, 2743 (1988).

[36] B. Ratra, Astrophys. J. 391, L1 (1992).

[37] M. Giovannini, String theory and fundamental interactions, edited by M. Gasperini and J. Maharana, Lecture Notes in Physics (Springer, Berlin/Heidelberg, 2007) (arXiv:astro-ph/0612378).

[38] J. Martin and J. Yokoyama, Journal of Cosmology and Astroparticle Physics 01, 025 (2008). 
[39] K. Subramanian, Astron. Nachr. 331, 1, 110 (2010).

[40] J. R. Shaw and A. Lewis, Phys. Rev. D 81, 043517 (2010).

[41] I. A. Brown, ArXiv e-prints (2010), arXiv:1005.2982v1 [astroph.CO].

[42] G. Chen, P. Mukherjee, T. Kahniashvili, B. Ratra and Y. Wang, Astrophys. J. 611, 655 (2004).

[43] P. D. Naselsky, L-Y. Chiang, P. Olesen and O. V. Verkhodanov, Astrophys. J. 615, 45 (2004).

[44] A. Bernui and W.S. Hipolito-Ricaldi, Mon. Not. R. Astron. Soc. 389, 1453 (2008).

[45] T. Kahniashvili, G. Lavrelashvili and B. Ratra, Phys. Rev. D 78, 063012 (2008).

[46] L. Wang and M. Kamionkowski, Phys. Rev. D 61, 063504 (2000).

[47] E. Komatsu and D. N. Spergel, Phys. Rev. D 63, 063002 (2001).

[48] N. Bartolo, E. Komatsu, S. Matarrese and A. Riotto, Phys. Rep. 402, 3, 103266 (2004).

[49] J. R. Fergusson and E. P. S. Shellard, Phys. Rev. D 76, 083523 (2007).

[50] A. Riotto, The Quest for Non-Gaussianity, Lect. Notes Phys. 738, 305 (2008).

[51] A. P. S. Yadav and B. D. Wandelt, Advances in Astronomy 2010, 565248 (2010) (doi:10.1155/2010/565248).

[52] T. R. Seshadri and K. Subramanian, Phys. Rev. Lett. 103, 081303 (2009).
[53] C. Caprini, F. Finelli, D. Paoletti and A. Riotto, Journal of Cosmology and Astroparticle Physics 06, 021 (2009).

[54] R.-G. Cai, B. Hu and H.-B. Zhang, Journal of Cosmology and Astroparticle Physics 08, 025 (2010).

[55] I. Brown, and R. Crittenden, Phys. Rev. D 72, 063002 (2005).

[56] K. Kojima, T. Kajino and G. J. Mathews, Journal of Cosmology and Astroparticle Physics 02, 018 (2010).

[57] C. Bonvin and C. Caprini, Journal of Cosmology and Astroparticle Physics 05, 022 (2010).

[58] K. E. Kunze, ArXiv e-prints (2010), arXiv:1007.3163v1 [astroph.CO].

[59] K. Jedamzik, V. Katalinic and A. Olinto, Phys. Rev. D 57, 3264 (1998).

[60] K. Subramanian and J. D. Barrow, Phys.Rev. D 58083502 (1998).

[61] K. Subramanian, T. R. Seshadri and J. D. Barrow, Mon. Not. Roy. Astr. Soc. 344, L31 (2003).

[62] C. Caprini, R. Durrer, Phys. Rev. D 65, 023517 (2002).

[63] M. Giovannini, Phys. Rev. D 81, 127302 (2010).

[64] D. H. Lyth and A. R. Liddle, The Primordial Density Perturbation (Cambridge University Press, Cambridge U.K., 2009).

[65] M. Giovannini, PMC Physics A, 1:5 (2007) (doi:10.1186/17540410-1-5).

[66] I. S. Gradshteyn and I. M. Ryzhik, Table of Integrals, Series and Products (Academic Press, New York, U.S.A. and London U.K., 6th edition, 2000).

[67] E. Komatsu et al.,ArXiv e-prints (2010), arXiv1001.4538 [astro-ph.CO]. 